\title{
Impacto de la educación alimentaria y actividad física en la certificación de trabajador saludable
}

\author{
Impact of nutrition education and physical activity in healthy worker certification
}

Segundo Rivera Ruiz ${ }^{1}$, Walter Gómez Gonzales ${ }^{2}$

\section{RESUMEN}

Objetivo: Determinar el impacto de la educación alimentaria y actividad física en la certificación de trabajador saludable, en el Hospital I Tingo María ESSALUD, 2013.Materiales y Métodos: Estudio cuasiexperimental, en series de tiempo, con un grupo único, y dos mediciones: antes y después de la intervención: El diseño del estudio fue: $\mathrm{O} 1 \mathrm{X}$ O2 Donde (X) corresponde a la variable independiente (educación alimentaria y actividad física), la evaluación antes de la intervención medición ex ante - pre test (O1) y la evaluación después de la octava semana de intervención medición ex post - post test (O2). La población de estudio estuvo conformado por 75 trabajadores del Hospital Tingo María ESSALUD, que aceptaron participar en el estudio. Antes de la intervención y después de la intervención, se midió y consigno los valores de Glucosa, Presión Arterial, Colesterol, Triglicéridos, HDL, LDL e IMC, de los trabajadores incluidos en el estudio. Para procesar y analizar los datos se utilizó el software SPSS versión 21.0 para Windows y para el análisis de los datos y medir el nivel de asociación de las variables en estudio, la prueba T student. Resultados: El programa educación alimentaria y actividad física aplicado en $n=75$ trabajadores del Hospital I Tingo María durante un período de 08 semanas, permitió que 65 (87\%) aprobaran y pudieron acreditar valores normales para los indicadores considerados en el estudio, por tanto certificados como trabajadores saludables, mientras 10 trabajadores (13\%) no aprobaron y no accedieron a la certificación. Hubo un incremento de 08 puntos porcentuales (06 trabajadores) entre la primera y la segunda evaluación. Conclusión: El programa educación alimentaria y actividad física aplicado, tuvo efectos positivos en el incremento del número de trabajadores que accedieron a la certificación de trabajador saludable; obteniéndose una diferencia significativa en la prueba de hipótesis $\mathrm{t}=1,243$ para $\mathrm{p}<0,05$, respecto al control pretest, alcanzándose logros positivos en un promedio de $87 \%$ de casos.

PALABRA CLAVE: Educación alimentaria - Actividad física - Trabajador Saludable.

\begin{abstract}
Objective: To determine the impact of nutrition education and physical activity in healthy worker certification in Tingo Maria Hospital I ESSALUD, 2013.Material and Methods: A quasi-experimental study, time series, with one group, and two measurements: before and after the intervention: The study design was: Where O1 X O2 (X) is the independent variable (food education and physical) activity, before the measurement evaluation ex ante intervention - pre test $(\mathrm{O} 1)$ and evaluation after the eighth week of intervention measure ex post - post test (O2). The study population consisted of 75 workers Tingo María ESSALUD Hospital, who agreed to participate in the study. Preoperatively and postoperatively, and consign measured values of glucose, blood pressure, cholesterol, triglycerides, HDL, LDL and BMI, workers included in the study. To process and analyze the data SPSS software version 21.0 for Windows and for analyzing the data and measure the level of association of the variables under study, student $\mathrm{T}$ test was used. Results: The nutritional education and physical activity program implemented in $\mathrm{n}$ = 75 workers I Tingo María Hospital for a period of 08 weeks, allowed 65 (87\%) approve and could prove normal values for the indicators considered in the study therefore certified as healthy workers, while 10 workers $(13 \%)$ not approved and not agreed to certification. There was an increase of 08 percentage points (06 workers) between the first and the second evaluation. Conclusion: nutritional education and physical activity program implemented, had a positive impact on increasing the number of workers who agreed to the healthy worker certification; obtaining a significant difference in the hypothesis test $t=1,243$ for $\mathrm{p}<0.05$, compared to pretest control positive achievements in reaching an average of $87 \%$ of cases.
\end{abstract}

KEYWORDS: Food Education - Physical Activity - Healthy Worker.

\footnotetext{
${ }^{1}$ Médico Internista. Hospital Tingo María, EsSalud, Huánuco, Perú.

${ }^{2}$ Doctor en Ciencias de la Salud. Seguro Social de Salud - ESSALUD

Profesor Universidad María Auxiliadora - Lima 36, Perú.
} 


\section{INTRODUCCION}

La OPS manifestó que "los años de vida ajustados (AVAD) en función de la discapacidad y perdidos debido a enfermedades no transmisibles como obesidad, accidentes cerebro vasculares, diabetes y cardiopatías en las Américas ascienden a 12.5 millones de AVAD, que se suman a los 4.6 millones de AVAD perdidos debido a la desnutrición de niños y madres". Varias encuestas nacionales de América Latina y el Caribe muestran que entre un 50 y un $60 \%$ de los adultos tienen un peso excesivo o son obesos. "Entre los niños menores de 5 años, entre un 7 y un 12\% son obesos", es más, en México y Chile, las encuestas nacionales recientes indican que cerca del $15 \%$ de los adolescentes son obesos" $(1,2,3)$.

Las organizaciones vinculadas a la salud pública como la OPS, en la prevención de enfermedades crónicas, y muy especialmente, en la promoción de la salud a través del ejercicio físico y de una dieta saludable, están a la búsqueda de adecuadas estrategias de intervención para tener el mayor impacto público posible. En este marco es que el presente proyecto tiene como propósito implementar, fortalecer y posicionar la Atención Primaria de la Salud, cuyo modelo presenta una débil implantación en los Hospitales Nivel I del Seguro Social de Salud, a partir de la certificación de dichos hospitales como saludables; basado en el cumplimiento de ciertos indicadores de los proveedores de servicios y/o clientes internos (IMC, presión arterial, colesterol. circunferencia de cintura, entre otros con valores normales).

La sociedad está en la era de las computadoras, la televisión, los juegos electrónicos. En los colegios, en el trabajo, realmente no se promueve el deporte como debieran, y la comida chatarra ha ido dominando la canasta familiar, no hay una determinación genuina para la promoción de estilos de vida saludables que combatan los fenómenos del sedentarismo, la obesidad y la mala alimentación, así como que tampoco hay un nivel de involucramiento fuerte de los propios trabajadores de salud, respecto a fortalecer el modelo de la Atención Primaria de la Salud por la persistencia del modelo biologista.Por tanto es importante lograr la certificación de los trabajadores del Hospital I Tingo María como trabajador saludable en una fase inicial, con la intervención proactiva de los clientes internos (trabajadores) y de esta manera ser modelos de la APS y al mismo tiempo contribuir a disminuir la prevalencia de las enfermedades crónicas en la población adscrita al Hospital I Tingo María ESSALUD, como son hipertensión, obesidad, hipercolesterolemia.

En el marco de lo descrito el objetivo general del estudio es determinar el impacto de la educación alimentaria y actividad física en la certificación de trabajador saludable, en el Hospital I Tingo María ESSALUD, 2013. Los objetivos específicos: Evaluar los indicadores de salud (Glucosa, Presión Arterial, Triglicéridos, HDL, LDL, IMC), antes de la intervención; aplicar educación alimentaria y actividad física a los trabajadores incluidos en el estudio, durante 08 semanas y finalmente establecer el impacto de la educación alimentaria y actividad física en la certificación de trabajador saludable, a partir de la precisión de las diferencias estadísticamente significativas entre las mediciones antes y después de la intervención, de los valores de los indicadores de salud como Glucosa, Presión Arterial, Triglicéridos, HDL, LDL, IMC.

\section{MATERIALES Y METODOS}

Diseño cuasi experimental con un grupo y dos mediciones antes y después de la intervención, seleccionado para el presente estudio, permite establecer una relación de causalidad de dos variables con un adecuado nivel de confiabilidad y validez, es el diseño que tiene el mayor poder explicativo cuando no se puede tener otro grupo de comparación y/o asignar al azar a los participantes.

\section{TIPO DE INVESTIGACION}

Es un estudio cuasiexperimental, en series de tiempo, con un grupo único, y dos mediciones: antes y después de la intervención.

Longitudinal, las variables sujetas a estudio se medirán en más de una ocasión para poder comparar los resultados obtenidos.

Prospectivo, se colectara la información observando el impacto de la variable independiente en el futuro.

El diagrama del estudio fue: $\mathbf{0 1} \times \mathbf{X} \quad \mathbf{0 2}$ 
Donde (X) corresponde a la variable independiente (educación alimentaria y actividad física), la evaluación antes de la intervención medición ex ante - pre test (O1), y la evaluación después de la octava semana de intervención medición ex post - post test $(\mathrm{O} 2)$.

\section{DISEÑO GRAFICO DEL ESTUDIO}

GRUPO DE ESTUDIO

TRABAJADORES DE SALUD - INGRESO AL ESTUDIO

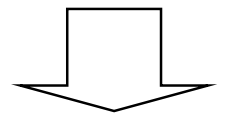

$\mathbf{O}_{1}$

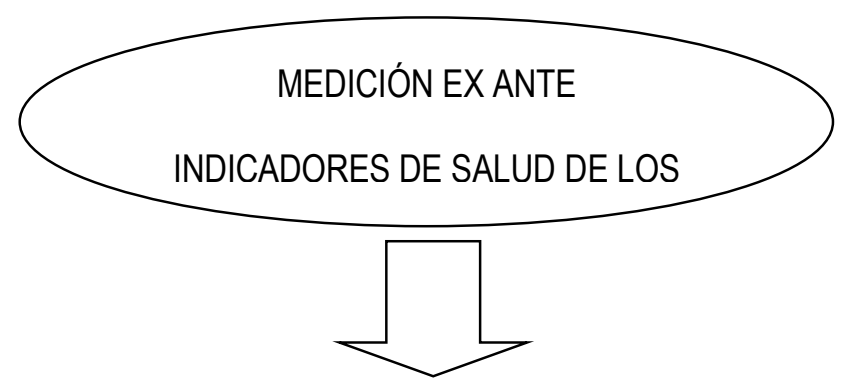

$\mathbf{x}$

INTERVENCIÓN EDUCACIÓN ALIMENTARIA Y ACTIVIDAD FÍSICA - DURACION 08 SEMANAS

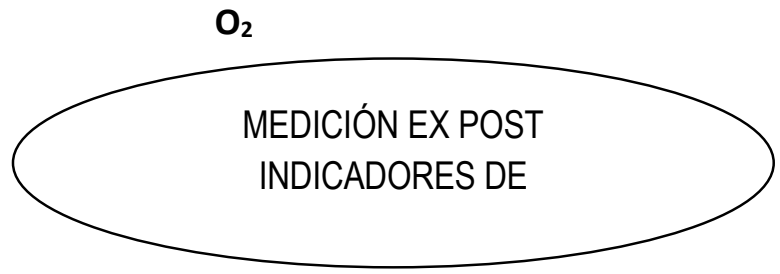

COMPARACIÓN IMPACTO DE LA INTERVENCIÓN

Dónde: $X=$ intervención

0 = Observación 


\section{POBLACION Y MUESTRA}

La población objeto de estudio estuvo conformado por los trabajadores del Hospital I Tingo María ESSALUD, que aceptaron participar en el estudio, siendo 75 los trabajadores incluidos.

\section{TAMAÑO MUESTRAL}

No fue pertinente realizar cálculo de tamaño muestral.

\section{CRITERIOS DE INCLUSIÓN Y EXCLUSION:}

\section{CRITERIOS DE INCLUSIÓN:}

$\checkmark$ Trabajador que tiene vínculo laboral con ESSALUD, por cualquier modalidad.

$\checkmark$ Asentimiento verbal de participación en el estudio.

\section{CRITERIOS DE EXCLUSIÓN:}

$\checkmark \quad$ Trabajador que no tiene vínculo laboral con ESSALUD, por cualquier modalidad.

$\checkmark \quad$ No Asentimiento verbal de participación en el estudio.

$\checkmark$ Trabajador que presenta alguna discapacidad que no permita realizar actividad física.

\section{PROCEDIMIENTOS}

El diseño del estudio se ha realizado con el criterio de conformar y contribuir a la certificación de un trabajador saludable, a través de realizar una intervención consistente en procesos interactivos de alimentación saludable y actividad física durante 08 semanas consecutivas que permitan mejorar los indicadores de certificación, previo a la intervención (evaluación ex ante intervención y/o basal), para luego volver a medir los mismos indicadores (evaluación ex post y/o de impacto, los trabajadores incluidos en el estudio para certificar deben de acreditar valores normales en los indicadores, caso contrario no certifica y debe de volver a repetir al proceso hasta lograr la certificación debiendo ser un proceso proactivo de parte de los trabajadores, acto que permitirá más adelante lograr incentivos cualitativos y/o cuantitativos a los trabajadores del
Hospital que certifiquen con los indicadores incluidos en el estudio luego de la intervención.

\section{INTERVENCIÓN REALIZADA}

\section{EDUCACIÓN EN ALIMENTACIÓN \\ SALUDABLE}

Demostraciones de dietas saludables.

Consumo de alimentos saludables (frutas).

Trípticos, mosquitos.

\section{ACTIVIDAD FISICA}

Caminata diaria 30 minutos 03 veces a la semana y/o otra actividad física que practique el trabajador.

- DURACIÓN: 08 semanas.

\section{TECNICAS APLICADAS EN LA RECOLECCION DE LA INFORMACION}

$\checkmark$ Ficha Medico Ocupacional, para consignar los datos de ingreso antes de la intervención y resultado después de la intervención, respecto a Glucosa, Presión Arterial, Colesterol, Triglicéridos, HDL, LDL e IMC, de los trabajadores incluidos en el estudio

$\checkmark$ Examen bioquímico de sangre - valores de ingreso y resultado de triglicéridos, colesterol, HDL, LDL y glucosa.

\section{PROCESAMIENTO DE DATOS:}

Para procesar los datos se utilizo el software SPSS versión 16.0 para Windows y para el análisis de los datos y medir el nivel de asociación de las variables en estudio, la prueba T student. Se aceptara diferencias estadísticamente significativas un valor $\mathrm{p}<0.05$.

\section{INSTRUMENTOS DE MEDICION}

$\begin{array}{ll}\checkmark & \text { Espectrofotómetro StatCal } \\ \checkmark & \text { Tensiómetro } \\ \checkmark & \text { Estetoscopio } \\ \checkmark & \text { Balanza con tallímetro estandarizado } \\ \checkmark & \text { Centímetro }\end{array}$




\section{RESULTADOS}

\section{CUADRO 01 - MEDICIÓN BASAL PROYECTO CTS}

EVALUACIÓN ANTES DE LA INTERVENCIÓN

\begin{tabular}{|l|c|c|}
\hline \multicolumn{1}{|c|}{ EVALUACIÓN } & № & \% \\
\hline APROBADOS & 59 & 79 \\
\hline DESAPROBADOS & 16 & 21 \\
\hline TOTAL & 75 & 100 \\
\hline
\end{tabular}

Fuente: Proyecto CTS

El grafico 01 nos muestra los resultados de la evaluación ex ante de los trabajadores del Hospital I Tingo María ESSALUD, hallándose de 75 trabajadores incluidos en el estudio a 59
(79\%) que aprobaron y pudieron acreditar valores normales para los indicadores considerados en el estudio, mientras 16 trabajadores $(21 \%)$ no aprobaron.

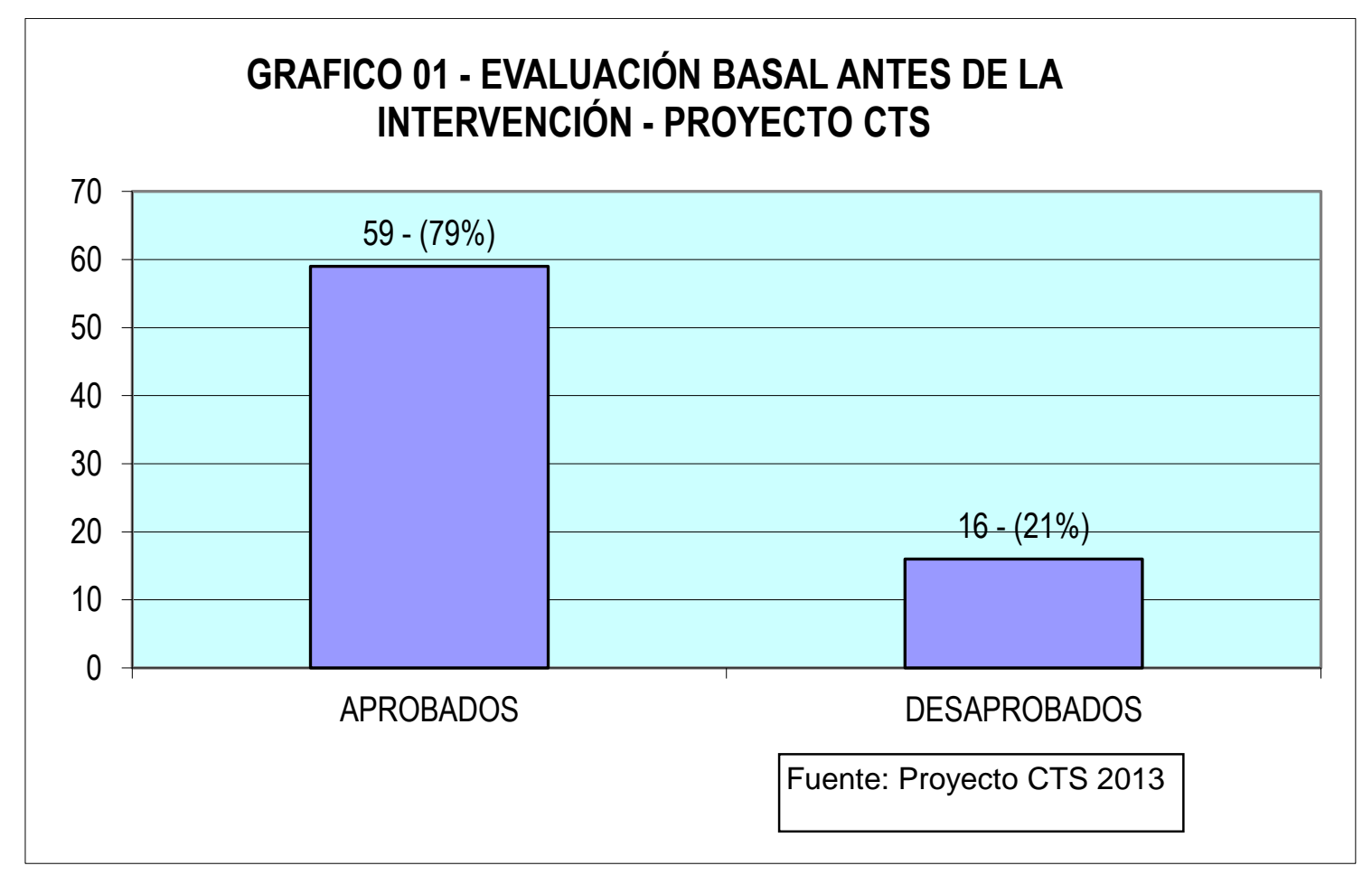


El grafico 02 nos muestra los resultados de la evaluación ex post de los trabajadores del Hospital I Tingo María ESSALUD, hallándose de 75 trabajadores incluidos en el estudio a 65
(87\%) que aprobaron y pudieron acreditar valores normales para los indicadores considerados en el estudio, mientras 10 trabajadores $(13 \%)$ no aprobaron.

\section{CUADRO 02 - MEDICIÓN DE INDICADORES PROYECTO CTS}

EVALUACIÓN DESPUES DE LA INTERVENCIÓN

\begin{tabular}{|l|c|c|}
\hline \multicolumn{1}{|c|}{ EVALUACIÓN } & $\mathbf{N}^{\mathbf{0}}$ & $\boldsymbol{\%}$ \\
\hline APROBADOS & 65 & 87 \\
\hline DESAPROBADOS & 10 & 13 \\
\hline TOTAL & $\mathbf{7 5}$ & $\mathbf{1 0 0}$ \\
\hline
\end{tabular}

Fuente: Proyecto CTS

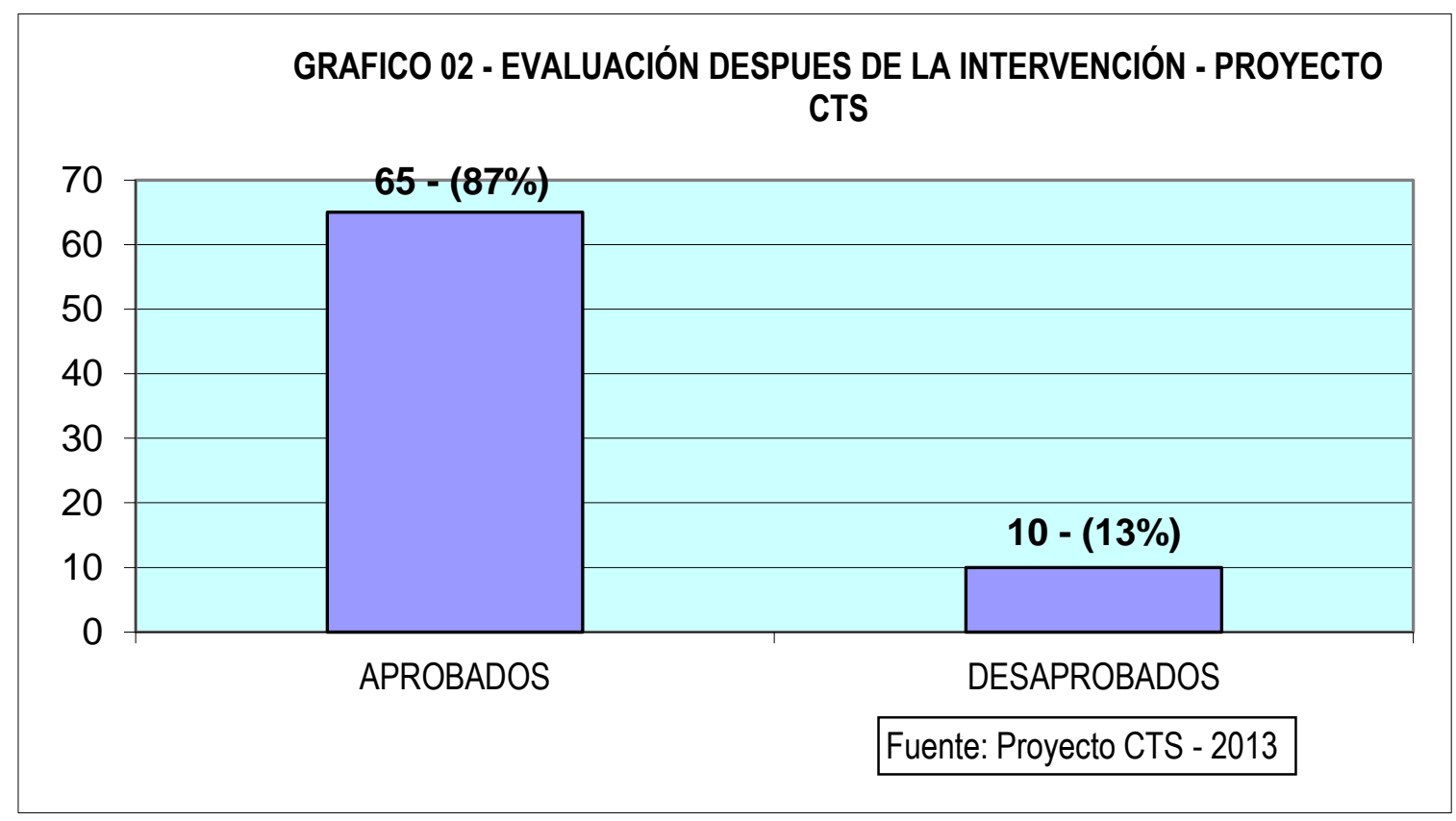

El grafico 03 nos muestra los resultados en sentido comparativo, de la evaluación ex ante intervención versus la evaluación ex post intervención de los trabajadores del Hospital I Tingo maría ESSALUD, en base a los indicadores propuestos y a los umbrales de calidad aceptados para la certificación, hallándose como resultado de la intervención de 75 trabajadores incluidos en el estudio a $65(87 \%)$ que aprobaron y pudieron acreditar valores normales para los indicadores 
considerados en el estudio, por tanto certificados como trabajadores saludables, mientras 10 trabajadores (13\%) no aprobaron y no accedieron a la certificación como trabajador saludable.

Existe un incremento de 08 puntos porcentuales (06 trabajadores) entre la primera y la segunda evaluación, sin embargo debemos de manifestar que aún hay trabajadores que no participaron en el estudio, entre personal asistencial y administrativo, así como trabajadores que no pudieron acreditar los valores normales establecidos para este fin, habiéndose logrado luego de la intervención llegar a un $87 \%$ de trabajadores del total de trabajadores incluidos en el estudio, por lo explicitado en nuestro Hospital, es necesario repetir el ciclo de mejora hasta llegar al cumplimiento de los indicadores propuestos, debiendo ser este un compromiso institucional y de los directivos el certificar a por lo menos el 90\% del total de trabajadores del Hospital como trabajador saludable, como estrategia fundamental para el posicionamiento adecuado de la Atención Primaria de la Salud en el Seguro Social de Salud, en el marco del fortalecimiento del nuevo modelo de Atención Integral de Salud.

\section{CUADRO 03 - COMPARATIVO MEDICIÓN EX ANTE VERSUS MEDICIÓN EX POST PROYECTO CTS - 2013}

\begin{tabular}{|l|c|c|c|c|}
\hline \multirow{2}{*}{$\begin{array}{c}\text { EVALUACIÓN DE } \\
\text { INDICADORES }\end{array}$} & \multicolumn{2}{|c|}{ EVALUACIÓN EX ANTE } & \multicolumn{2}{c|}{$\begin{array}{c}\text { EVALUACIÓN EX } \\
\text { POST }\end{array}$} \\
\cline { 2 - 5 } & $\mathbf{N}^{\mathbf{0}}$ & $\boldsymbol{\%}$ & $\mathbf{N}^{\mathbf{o}}$ & $\boldsymbol{\%}$ \\
\hline APROBADOS & 59 & 79 & 65 & 87 \\
\hline DESAPROBADOS & 16 & 21 & 10 & 13 \\
\hline TOTAL & $\mathbf{7 5}$ & $\mathbf{1 0 0}$ & $\mathbf{7 5}$ & $\mathbf{1 0 0}$ \\
\hline
\end{tabular}

Fuente: Proyecto CTS

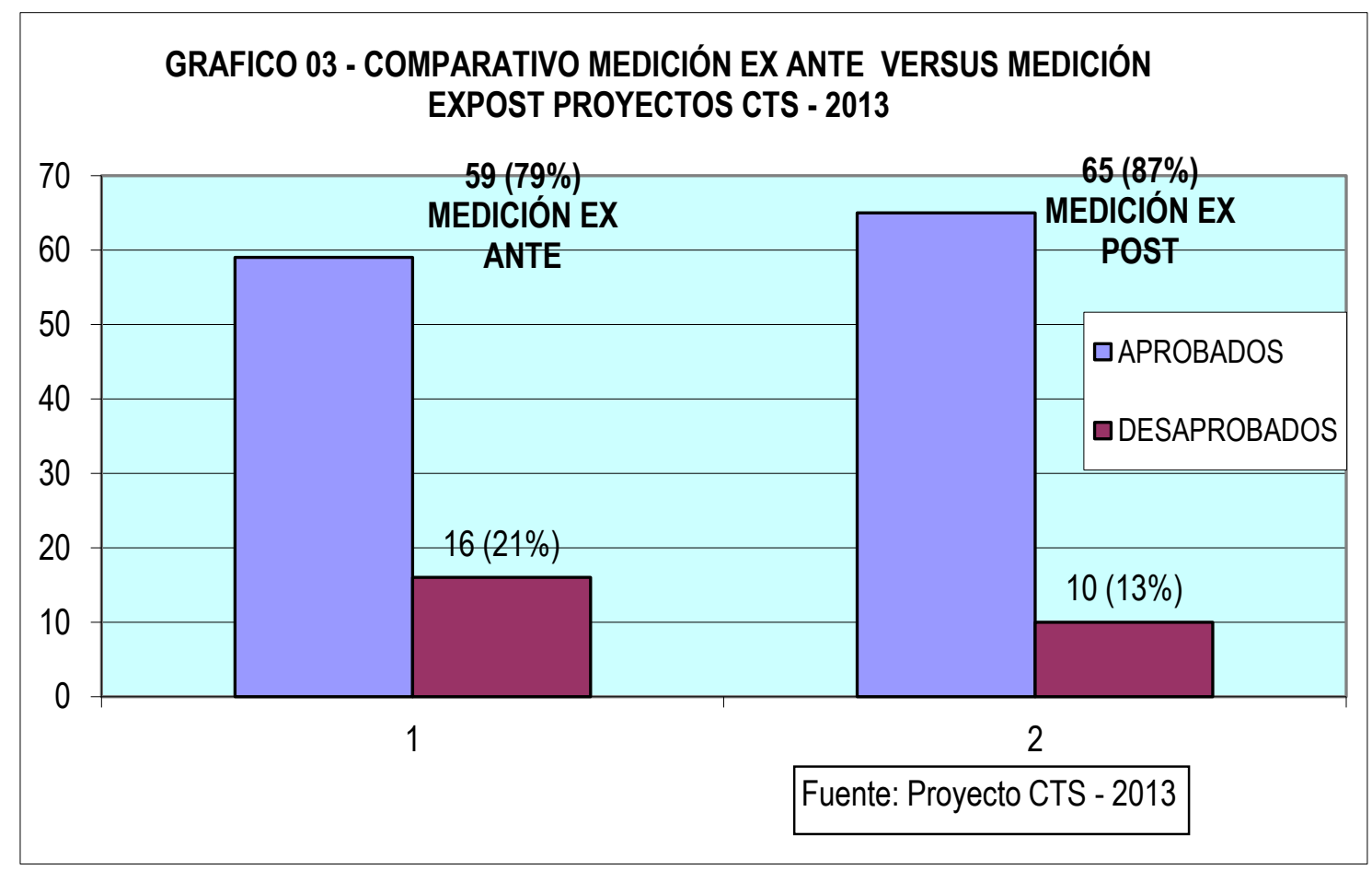




\section{EVALUACIÓN Y RESULTADOS DEL IMPACTO}

La variación promedio de resultados de impacto del programa educación alimentaria y actividad física, respecto al pre test, se evaluó estadísticamente aplicándose la prueba de hipótesis bilateral " $t$ ", cuyos valores estadísticos de comparación son los siguientes:

\begin{tabular}{|l|l|}
\hline Pre test & Post test \\
\hline $\mathrm{na}=75$ & $\mathrm{nb}=75$ \\
$\overline{\mathrm{X}} 1=0,59$ & $\overline{\mathrm{X} 2}=0,65$ \\
$\mathrm{~S} 1=0,40$ & $\mathrm{~S} 2=0,22$ \\
& \\
\hline
\end{tabular}

Reemplazando valores para el error standard se tiene:

$$
\begin{aligned}
& \mathrm{EEa}=0,10 \\
& \mathrm{EEb}=\frac{0,22}{\sqrt{75}}=0,055
\end{aligned}
$$

Sustituyendo valores para " $\mathrm{t}$ ":

$$
\mathrm{t}=\frac{0,59-0,65}{\sqrt{0,10^{2}+0,09^{2}}}=1,243
$$

$\mathrm{tc}=1,243, \mathrm{~g} \ell=30$

$\mathrm{tT}= \pm 2,750, \alpha=0,05$

Obteniéndose un resultado según " $\mathrm{t}$ " tabular, significativo.

Según el análisis estadístico respectivo, el programa educación alimentaria y actividad física aplicado en $\mathrm{n}=75$ trabajadores del Hospital I Tingo María durante un períodos de 08 semanas, tuvo efectos positivos en el cambio de actitudes, practicas, adquirir y aplicar conocimientos sobre el educación alimentaria y actividad física; obteniéndose una diferencia significativa en la prueba de hipótesis $\mathrm{t}=1,243$ para $\mathrm{p}<0,05$, respecto al control pre test, alcanzándose logros positivos en un promedio del $87 \%$ de los casos.

\section{DISCUSION}

El análisis estadístico del estudio muestra que el programa de educación alimentaria y actividad física aplicado en los trabajadores del Hospital I Tingo María, durante un período de 08 semanas, tuvo efectos positivos en el cambio de actitudes, prácticas, adquirir y aplicar conocimientos sobre educación alimentaria y actividad física. Estos hallazgos son consistentes con el estudio realizado Sanabria-Ferrand, González Q., Urrego D. (14), sobre estilos de vida saludable en profesionales de la salud colombianos, estableciendo que si existe alguna relación entre su nivel de acuerdo con el modelo biomédico y su estilo de vida, así también encontró que sólo el $11,5 \%$ de los médicos y el $6,73 \%$ de las enfermeras presentan un estilo de vida saludable y que el principal problema está relacionado con la actividad física y el deporte; por otro lado también nuestros hallazgos son coincidentes con informes emitidos por la Women's Healt Weekly (15), acerca de un Programa innovador de Salud Materna en las comunidades de Guatemala, consistente en educación, información sobre la salud materna y organización para la situaciones de emergencia, cuyos resultados demuestran mejoras significativas en conocimiento, actitudes y prácticas entre hombres y mujeres expuestos al programa. Por otro lado según la Teoría de la Conducta Planificada de Ajzen (16), la conducta de una persona está determinada por la intención de realizarla, y ésta por la actitud hacia la conducta, la norma subjetiva y el control de la conducta percibida.

En este sentido, el proyecto logró cambios significativos en los conocimientos y actitudes de los trabajadores respecto a la alimentación saludable y actividad física, asimismo, Hawkins, Joellen W.; Aber, Cynthia S.; Cannan, Alice; Coppinger, Christine M.; Rafferty, Kathleen O'Brien (17), en su estudio sobre el auto cuidado de la mujer embarazada, mencionan que la responsabilidad de las conductas de autocuidado son influenciadas por el deseo de la madre de tener un embarazo saludable, los proveedores de salud y la red social, que si bien es cierto no es una intervención vinculada a actividad física y/o alimentación saludable, es menester citarlo porque tiene sustento sobre las conductas de autocuidado de las personas, como es el caso de los trabajadores de salud. 


\section{CONCLUSION}

El programa educación alimentaria y actividad física aplicado, tuvo efectos positivos en el incremento del número de trabajadores que accedieron a la certificación de trabajador saludable; obteniéndose una diferencia significativa en la prueba de hipótesis $t=1,243$ para $\mathrm{p}<0,05$, respecto al control pretest, alcanzándose logros positivos en un promedio de $78 \%$ de casos.

Replicar y socializar la intervención realizada, adecuándolo siempre a la realidad local e incluir el método de investigación e intervención

Es importante darle a los programas un enfoque holístico que abarque la totalidad del ciclo vital y en la que se tenga en cuenta los determinantes de la salud y se haga hincapié en un proceso continuo de prevención y promoción de la salud, que permitan a los trabajadores y sus familiares mantenerse sanas y productivas.

Implementar el ciclo de mejora continua del proyecto CST en el Hospital I Tingo María, así como en otros establecimientos de salud del primer nivel de atención de salud de la red de CAS del Seguro social de salud.

\section{AGRADECIMIENTOS}

Un especial agradecimiento al personal del Hospital Tingo María Essalud, quienes participaron en la ejecución del estudio.

\section{AUTOR DE CORRESPONDENCIA:}

Walter E. Gómez, Seguro Social de Salud ESSALUD.

Profesor Universidad María Auxiliadora,

Av. Canto Bello 431- Ciudad Universitaria, Lima 36 - Perú.

Teléfono: +51-1-3891212.

E-mail:waltergomez29@yahoo.com

\section{REFERENCIAS BIBLIOGRAFICAS}

1. La Organización Mundial de la Salud (OMS)

2. Conferencia Internacional sobre Promoción de la Salud. Carta de Ottawa para la Promoción de la Salud. Ottawa, Ontario, Canadá: Organización Mundial de la Salud; Ministerio de Salud y Bienestar Social del Canadá y la
Asociación Canadiense de Salud Pública, 1986.

OPS. 2000. "La Salud de las Américas"

3. Resolución de la Asamblea Mundial de la Salud WHA57.17, 2004. Estrategia mundial sobre régimen alimentario, actividad física y salud. $57^{\mathrm{a}}$ Asamblea Mundial de la Salud.

4. Sanabria-Ferrand, González Q., Urrego D., 2007. Estilos de vida saludable en profesionales de la salud colombianos. Revista Med.15 (2): 207-217.

5. Manrique Peñaloza, Rosalinda. 2004. Los factores sociales de riesgo que influyen en los estilos de vida de la población trabajadora de Essalud.

6. Borjas Pezo, Hernán. 1995. Estudio sobre "Ejercicios terapéuticos como alternativa para el control no farmacológico de la hipertensión arterial".

7. Resolución de la Asamblea Mundial de la Salud WHA57.17, 2004. Estrategia mundial sobre régimen alimentario, actividad física y salud. 57 ${ }^{\mathrm{a}}$ Asamblea Mundial de la Salud.

8. World Health Organization (2002). The World Health Report 2002: Reducing Risks, Promoting Healthy Life. Geneva, World Health Organization.

9. Diet, Nutrition and Prevention of chronic diseases. Report of a Joint WHO/FAO Expert Consultation. World Health Organization. Geneva 2003. WHO Technical Report series 916.

10. Estrategia NAOS. Invertir la tendencia de la obesidad. Estrategia para la nutrición, actividad física y prevención de la obesidad. Madrid: Agencia Española de Seguridad Alimentaría. Ministerio de Sanidad y Consumo; 2005.

11. Artículos de revisión. Bases científicas de una alimentación saludable. rev med univ navarra/vol 50, no 4, 2006, 7-14

12. Tuomilehto J. Cardiovascular risk: prevention and treatment of the metabolic syndrome. Diabetes Res Clin Pract. 2005;68 Suppl 2:S28-35.

13. Office of Surgeon General.. Physical activity and the health: a report of the surgeon general. Washington DC: US Department of Health and Human Services, Centers for Disease Control and Prevention; 1996. 
14. Office of Surgeon General.. Physical activity and the health: a report of the surgeon general. Washington DC: US Department of Health and Human ervices, Centers for Disease Control and Prevention; 1996.

15. Women's Health (2004). Program teaches Guatemalan communities to improve maternal survival. Women's ealth Weekly 8/12/2004, p114, 2p. Search.epnet.com.catalog.llu.edu.

16. Ajzen(1989). Perceived Behavioral Control. University of Massachusetts Amherst. Recuperado el 07 de diciembre de 2005 del sitio web: http://wwwunix.oit.umass.edu/ aizen/pbc.html.

17. Hawkins, Joellen W.; Aber, Cynthia S.; Cannan, Alice; Coppinger, Christine M.; Rafferty, Kathleen O'Brien (1198). Women's Reported Self-Care Behaviors During Pregnancy. Health Care for
Women International, Nov/Dec98, Vol. 19 Issue 6, p529, 10p. Search.epnet.com.catalog.llu.edu. 18.

18. Declaración de Jakarta sobre la Promoción de la salud en el siglo XXI (1997). OPS/OMS Planificación local participativa: metodologías para la promoción de la salud en América latina y el Caribe. División de Promoción y Protección de la Salud, Washington, DC, 1999

19. Website:

www.paho.org/Spanish/HPP/5thGlobalC onf.htm.

20. Restrepo, Helena. Conceptos y definiciones. En: Promoción de la Salud: cómo construir vida saludable. Editorial Médica Panamericana, Bogotá, 2001: 2433.

Recibido: 07/04/2015

Aceptado: 27/04/2015 